\title{
An infrared coagulator to assist surgical treatment of infective endocarditis
}

\author{
Hiroshi Kubota $^{1}$, Hidehito Endo ${ }^{1}$, Hikaru Ishii ${ }^{1}$, Sachito Minegishi ${ }^{1}$, Hiroshi Tsuchiya ${ }^{1}$, Yu Takahashi ${ }^{2}$, \\ Satoko Funata ${ }^{1}$, Yusuke Inaba ${ }^{1}$ \\ ${ }^{1}$ Department of Cardiovascular Surgery, Kyorin University, Tokyo, Japan; ${ }^{2}$ Department of Cardiovascular Surgery, National Disaster Medical Center, \\ Tokyo, Japan \\ Correspondence to: Hiroshi Kubota. Department of Cardiovascular Surgery, Kyorin University, Tokyo, Japan. Email: kub@ks.kyorin-u.ac.jp.
}

Submitted May 14, 2019. Accepted for publication Sep 09, 2019.

doi: $10.21037 /$ jtd.2019.09.82

View this article at: http://dx.doi.org/10.21037/jtd.2019.09.82

We previously demonstrated that infrared coagulator IRK-151 (Lumatec, Deisenhofen, Germany) is capable of creating a transmural lesion while preserving the epicardium and endocardium of the beating right atrium and right ventricle in a canine model (1). Since the original IRK-151 model contains a 3-sec timer and its plastic body is not strong enough to tolerate long coagulation times, we modified it by replacing the body with reinforced heatresistant plastic and substituting a digital programmable timer so that it would be strong enough to perform myocardial ablation, and we demonstrated that the coagulator is capable of creating a conduction block on the beating right atrium clinically (2).

\section{Technology and technique}

We named the newly developed coagulator "Kyo-co (Kyorin-coagulator: Photon incorporation, Saitama, Japan)." Coagulator "Kyo-co" is sufficiently heat resistant to enable long and repeated application of infrared energy to tissue, to create deep lesions in the myocardium. A reflector in the infrared coagulator focuses light from a tungstenhalogen lamp into a light-conducting $10-\mathrm{mm}$ diameter quartz rod, and it emerges as $35 \mathrm{~W} / \mathrm{cm}^{2}$ of near-infrared light energy (wavelength, 400 to approximately 1,600 nm; peak wavelength, $850 \mathrm{~nm}$ ). Power output, ablation time, interval time, and total number of applications can be adjusted as needed, and the temperature of the tip is monitored to prevent overheating. Maximum ablation time is limited to 90 seconds. These parameters are shown on a digital display panel (Figure 1). Our preliminary experiment showed that maximum tissue temperature rose to $97.9+2.1^{\circ} \mathrm{C}$ for a total of $28 \mathrm{~s}(4 \mathrm{~s} \times 5$ times at 2 -sec intervals) (Figure 2). No medical infrared ablation system has ever tolerated such long and repeated use.

Histopathological examination of the ablated myocardium revealed well-demarcated transmural photocoagulation necrosis without carbonization or vaporization. It was possible to create a lesion to a maximum depth of $10.3 \mathrm{~mm}$ in the canine right ventricle lesion could made (Figure $3 A$ ). The myocardium did not revive in the chronic phase after the ablation. Hemosiderin deposition, macrophage invasion, increased capillary vessels, and increased juvenile collagen fibers were observed. The endocardium was thickened (Figure 3B,C).

\section{Clinical experience}

After receiving the approval of the ethics committee of Kyorin University to conduct a clinical and epidemiologic study entitled "surgical treatment of arrbytbmias, infectious endocarditis, and cardiac tumors with an infrared coagulator", written consent was obtained from the patient to perform the operation described below.

A 62-year-old male was admitted to our hospital with chief complaints of fever of unknown origin, arthralgia and melena. No evidence of heart failure was observed. Blood tests showed a leukocyte count of 33,600/hpf and C-reactive protein level of $33.0 \mathrm{mg} / \mathrm{dL}$. Computed tomography revealed splenic infarction, asymptomatic brain infarction, and cholecystitis. Ultrasound cardiography and computed tomography showed severe aortic valve stenosis due to a 

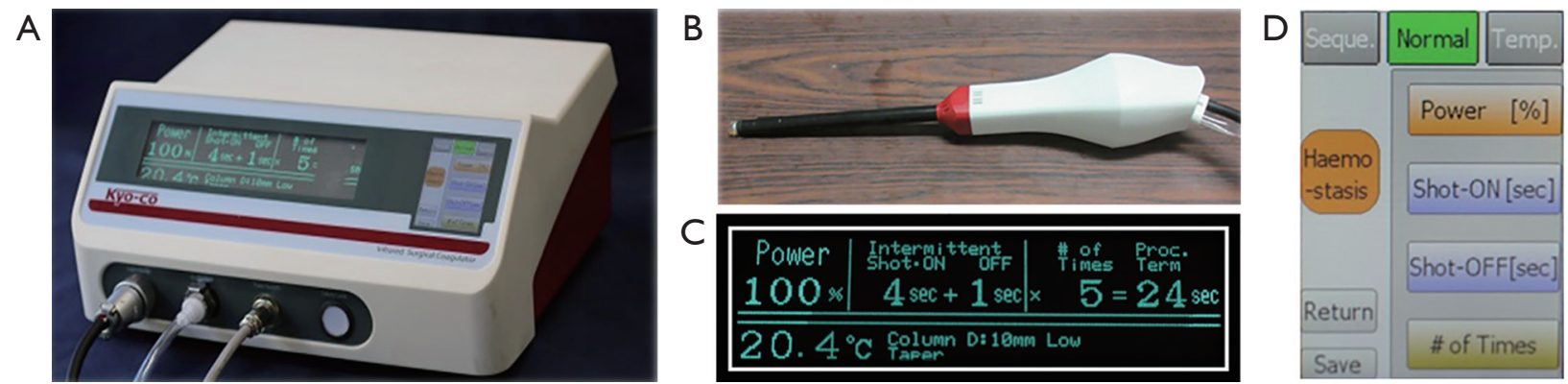

Figure 1 Infrared coagulator "Kyo-co". (A) The body of the coagulator is connected to the light-guide and foot switch. The vacuum system in the body is connected to the light-guide to cool down; (B) light-guide reinforced with heat-resistant plastic. Light from a tungstenhalogen lamp emerges as $35 \mathrm{~W} / \mathrm{cm}^{2}$ of near-infrared light energy (wavelength: 400 to approximately 1,600 nm; peak wavelength: $850 \mathrm{~nm}$ ); (C,D) digital programmable panel. Power, ablation time, interval time, total number of applications, and tip temperature are displayed.

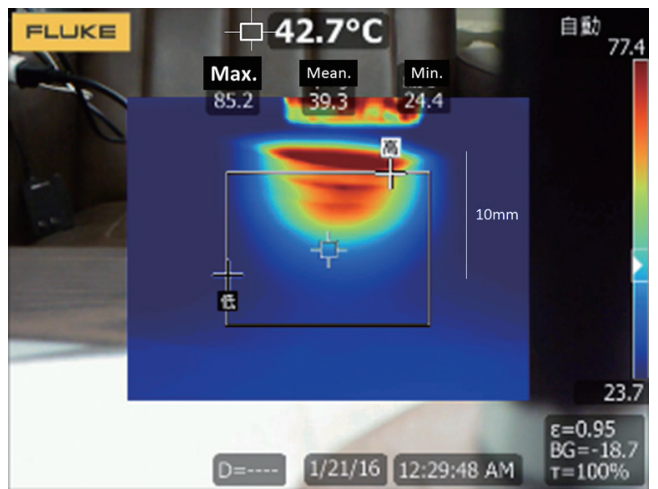

Figure 2 Preliminary experiment on chicken muscle tissue to measure the temperature. A cone-shaped high temperature lesion was observed. The maximum temperature was raised to $97.9+2.1^{\circ} \mathrm{C}$.

bicuspid valve (aortic valve area: $0.88 \mathrm{~cm}^{2}$, max pressure gradient: $76 \mathrm{mmHg}$ ) with vegetations and two large aortic annular abscesses (Figure 4). Ejection fraction was maintained (71\%). The diameter of the ascending aorta was $48 \mathrm{~mm}$.

A diagnosis of IE and acute hemorrhagic cholecystitis was made. Percutaneous gallbladder drainage and consecutive urgent open cholecystectomy were performed. After confirming resolution of the melena, elective surgery consisting ascending aortic replacement, aortic root plasty, and aortic valve replacement was planned. The patient had a history of steroid administration for Vogt-Koyanagi-Harada disease. No evidence of diabetes mellitus was detected.

The operation was performed in July 2014. A cardiopulmonary bypass was established by bicaval and ascending aortic cannulation through median sternotomy.
There was a moderate amount of bloody pericardial effusion and moderate pericardial adhesion. When tympanic membrane temperature had fallen to $24^{\circ} \mathrm{C}$, the ascending aorta was opened under hypothermic circulatory arrest with intermittent pressure augmented retrograde cerebral perfusion. After performing a distal anastomosis using a Triplex $30 \mathrm{~mm}$ graft (Terumo, Tokyo, Japan), antegrade perfusion and warming were restored. The aortic valve was bicuspid. Two large annular abscess orifices (both: $15 \times 10 \mathrm{~mm})$ were identified. One orifice was located beneath the left coronary artery orifice and involved the left commissure, and the other orifice was located beneath the right coronary artery orifice and involved the right commissure. It was possible to insert a Kelly forceps deep inside both abscess cavities. The myocardial tissue of both orifices was edematous and fragile. Curettage was performed on the margin of both abscess orifices, but in order to prevent coronary artery injury and prevent complete aortoleft ventricular dehiscence we were careful not to perform excessive debridement, and thus, fragile inflammatory tissue around the orifices remained. Infrared ablation was applied to these fragile lesions. Six seconds of ablation at 2-sec intervals was repeated 3 to 5 times at one point until the fragile tissue became dry and discolored (Figure 5). After ablating the entire margins of the orifices of both abscess cavities, xenopericardial trimmed patches were attached with 5-0 polypropylene continuous sutures. Before closing the orifices, the abscess cavities were filled with biological glue (Beriplast P-combi set; CSL Behring, Marburg, Germany) containing the antibiotic Amikacin. Mechanical valve replacement was then performed. Half of the sutures were placed directly in the xenopericardial patches.

After proximal anastomosis of the graft, the aorta was 
A

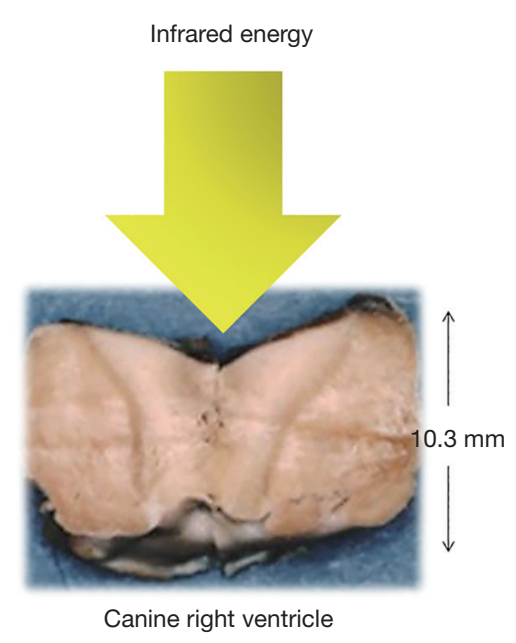

B

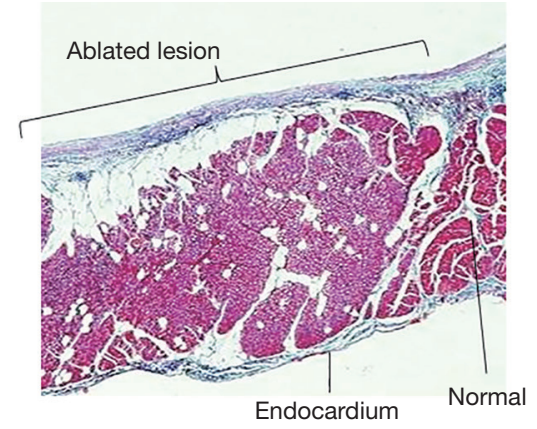

C

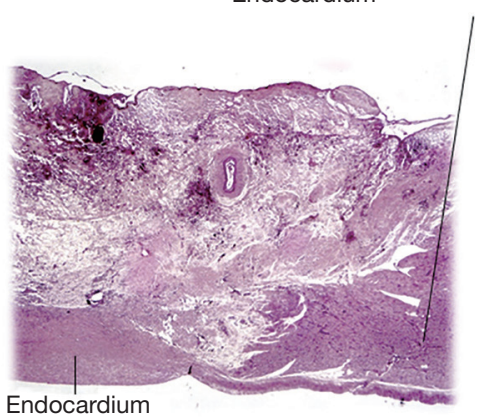

Figure 3 Histopathological change in the myocardium in a canine model. (A) It was possible to produce a transmural lesion having a of maximum depth of $10.3 \mathrm{~mm}$ in the canine right ventricle; (B) in an acute experiment, a well demarcated lesion was observed. Welldemarcated transmural photocoagulation necrosis without carbonization or vaporization was shown; AZAN, $\times 10$; (C) 3 months after the ablation, hemosiderin deposition, macrophage invasion, increased capillary vessels, and increased juvenile collagen fibers were observed. Hematoxylin-Eosin (HE), $\times 10$. The endocardium was thickened. Vessels were intact.
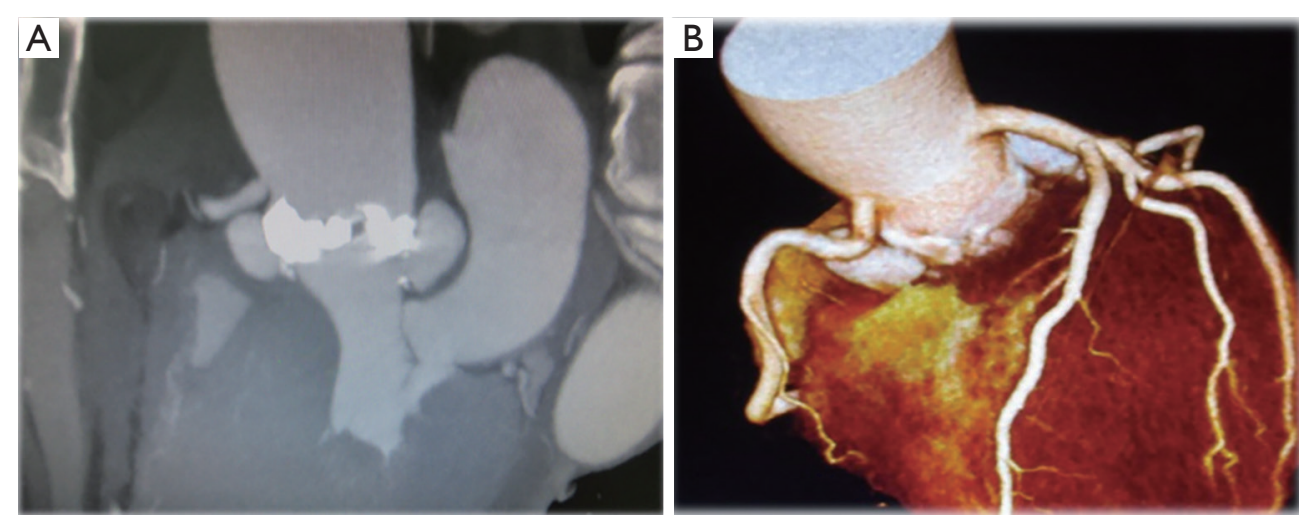

Figure 4 Preoperative computed tomography. (A) Two large aortic root abscesses were detected. The aortic valve is heavily calcified and the ascending aorta enlarged; (B) 3D image. Each abscess was located just beneath a coronary artery.

declamped, and weaning from the cardiopulmonary bypass was achieved without difficulty.

\section{Postoperative course}

Microscopic examination of the resected specimen revealed destruction of the endocardium and infiltration by pyogenic inflammatory cells.

Postoperative CT confirmed that both abscesses had diminished in size and no longer seen (Figure 6). The patient is alive and well 5 years after surgery without any signs of recurrence of the infection. 

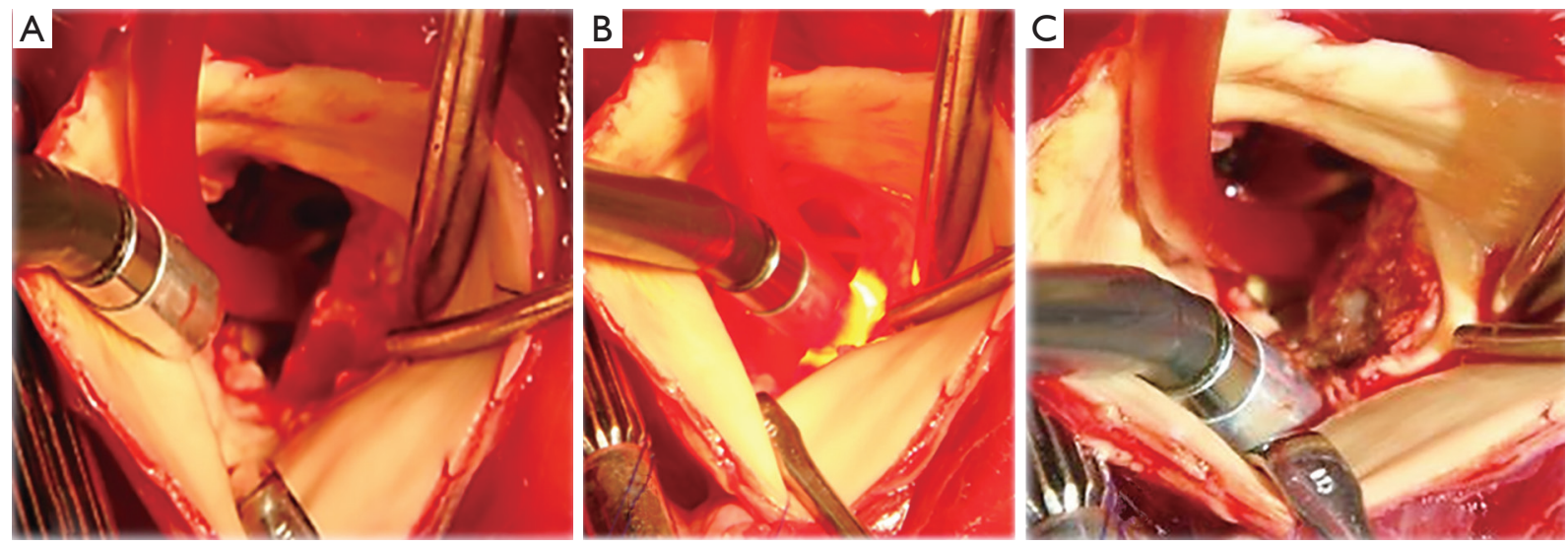

Figure 5 Intraoperative infrared ablation of the orifice of the abscess cavity. (A) Post-debridement, pre-ablative fragile annular tissue. Complete resection of the fragile tissue was impossible; (B) infrared ablation; (C) post-ablation. Discolored, sterilized annular tissue had become firm and provided us with a reliable suture line.

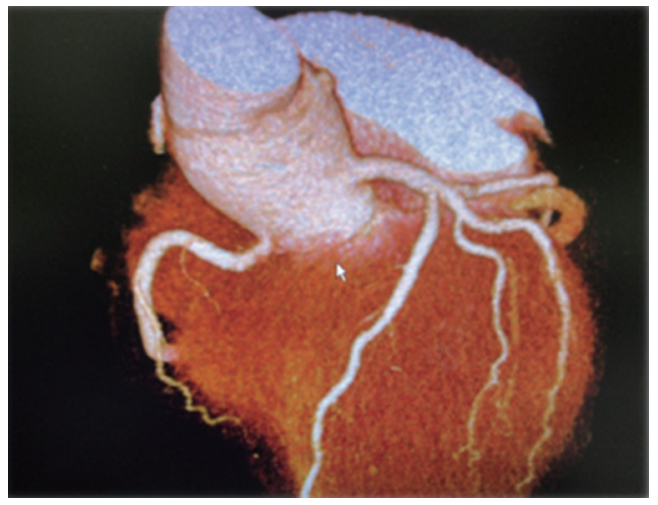

Figure 6 Computed tomography 1 month after the operation. Both abscess cavities had completely disappeared.

Thus far, 15 patients with IE involving aortic and/ or mitral annular lesion(s), including two patients with methicillin-resistant Staphylococcus aureus mitral valve endocarditis, one patient with methicillin-resistant Stapbylococcus aureus aortic valve endocarditis, and one patient with methicillin-resistant Staphylococcus aureus prosthetic mitral valve endocarditis, have been surgically treated with the assistance of infrared ablation. An annular lesion or annular lesions extended into the left ventricle in 3 patients, a fistula from the left ventricle to the right atrium was detected in 2 patients, and the mitro-aortic curtain was involved in one patient. The local recurrence rate was $0 \%$, and the complication rate related to the infrared ablation was $0 \%$. There were two deaths unrelated to the endocarditis: one due to non-occlusive mesenteric ischemia and the other due to perforation of the small intestine. None of the patients required re-operation during follow-up periods ranging from 0.4 to 5.4 years (mean: $1.8 \pm 1.3$ years) (Table 1).

\section{Comments}

We previously reported efficacy of the infrared coagulator in the treatment of atrial fibrillation in animal models and clinical cases (3-5). Nath et al. reported observing irreversible loss of cellular excitability and tissue injury after exposure to temperatures $>50{ }^{\circ} \mathrm{C}$ for 60 seconds (6). We applied this thermal effect as a means of achieving a bactericidal effect in the treatment of IE. High temperature short time (HTST) pasteurization is known as one of the pasteurization techniques. In HTST pasteurization, tissue temperatures between 72 and $75^{\circ} \mathrm{C}$ destroy pathogenic organisms including bacteria and viruses.

Intramyocardial abscess formation is usually caused by continuous spread of microorganism into the paravalvular myocardium, but the etiology of abscess formation is not restricted to continuous spread to the paravalvular myocardium. Sometimes, bacterial spillover into the blood leads to dissemination and proliferation at deeper sites and causes both abscess formation and tissue destruction (7). This is the theoretical background underlying the bactericidal effect of infrared energy in deeper lesions.

In the case reported here the myocardium was ablated intermittently at 2 -sec intervals, which prevents rapid temperature rises and charring. Charring not only blocks 


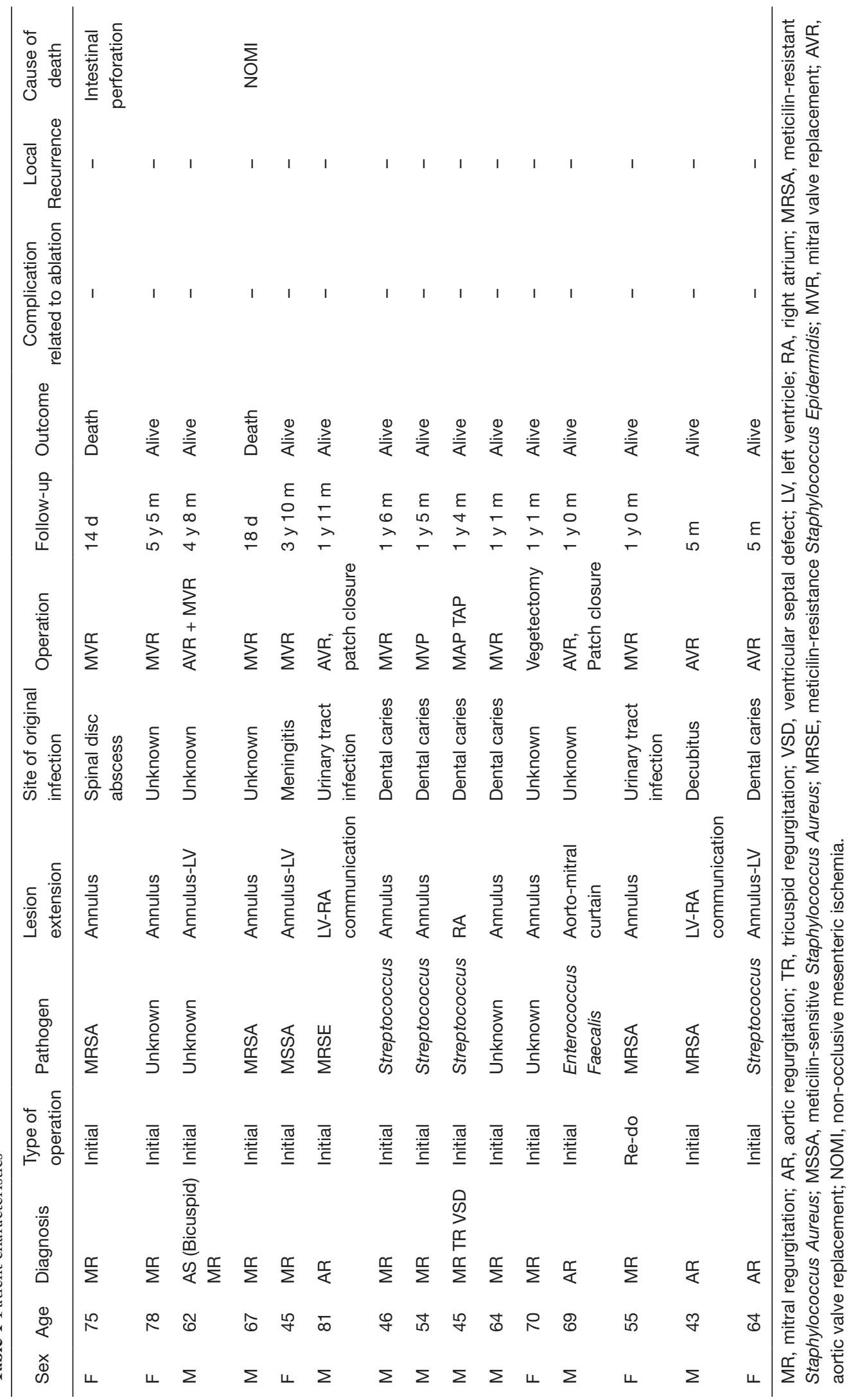


the photo-energy radiation deep inside the myocardium but is liable to provide a new infection site.

The coagulated edematous tissue in our patients became dry and firm, and it was possible to place more reliable sutures. Microscopically, the ablated myocardium was characterized by myocardial degeneration with karyopyknosis and atrophy of the cytoplasm. In the chronic phase, the endocardium thickened, and myocardium was totally replaced by collagen fibers. Collagen fibers endow tissue with elasticity, and they are very resistant to traction and tension forces. These are reasons why our patients showed good prognosis in long-term.

We have applied coagulator Kyo-co to both atria in 16 cases with atrial fibrillation without any complications (2). We have also applied coagulator Kyo-co to the whole infected mediastinal space concomitant with xenopericardial roll graft replacement in 9 cases to treat deep sternal wound infections with graft infection after ascending/arch aortic surgery (8). There was no recurrence of the infection in any of the cases. Eight patients are alive and well as of a followup period of 0.1 to 2.5 years. One patient died as a result of rupture of a thoracoabdominal aortic aneurysm.

We have also treated six cardiac myxoma patients by tumor resection with infrared ablation to the remaining tissue. Although one myxoma that originated in a mitral valve annulus could not be completely resected and the resected stump was tumor-cell positive, all patients are alive as of follow-up periods ranging from 0.1 to 7.9 years. One patient with metastatic cardiac liposarcoma to both ventricles who was treated surgically in assistant with infrared ablation died of local recurrence 15 months postoperatively.

The in-hospital IE mortality rate in the United States decreased after the 1960s and has remained stable high (19.7\%) (9). Since paravalvular abscess is an independent predictor of operative mortality in IE, and recurrent endocarditis is an independent predictor of death from all causes (10), our procedure may increase the efficacy of surgical treatment of IE.

\section{Limitations}

This study had some important limitations. This study was an observational study without assignment. There have been no controlled studies, and because it is difficult to procedure an animal model of IE, there have been no experimental studies.

\section{Conclusions}

It was possible to safely apply the infrared coagulator to infected tissue clinically without complications. Although the number of patients was small, an infrared coagulator "Kyo-co" may enable safe, more reliable, and less invasive treatment of IE.

\section{Acknowledgments}

The authors wish to thank Ms. Yuki Matsumoto for her assistance with the data analysis in this study.

Funding: This study was supported by funding from the "TERUMO Life Science Foundation, 2016" and "Leadingedge Industry Design Project, Medical Innovation, Saitama Prefecture, Japan, 2016".

\section{Footnote}

Conflicts of Interest: The authors have no conflicts of interest to declare.

Ethical Statement: The authors are accountable for all aspects of the work in ensuring that questions related to the accuracy or integrity of any part of the work are appropriately investigated and resolved. Written consent was obtained from the patient to perform the operation.

\section{References}

1. Kubota H, Furuse A, Takeshita M, et al. Atrial ablation with an IRK-151 infrared coagulator. Ann Thorac Surg 1998;66:95-100.

2. Kubota H, Endo H, Ishii H, et al. Epicardial infrared ablation to create a linear conduction block on a beating right atrium. J Cardiothorac Surg 2018;13:116.

3. Kubota H, Sudo K, Takamoto S, et al. Epicardial electrical isolation of the right atrial appendage on the beating heart with an infrared coagulator. Ann Thorac Surg 2009;87:1592-5.

4. Kubota H, Takamoto S, Takeshita M, et al. Atrial ablation using an IRK-151 infrared coagulator in canine model. J Cardiovasc Surg (Torino) 2000;41:835-47.

5. Kubota H, Takamoto S, Furuse A, et al. Epicardial Maze Procedure on the Beating Heart with an Infrared Coagulator. Ann Thorac Surg 2005;80:1081-6.

6. Nath S, Lynch C 3rd, Whayne JG, et al. Cellular 
electrophysiological effects of hyperthermia on isolated guinea pig papillary muscle. Implications for catheter ablation. Circulation 1993;88:1826-31.

7. Müller-Höcker J, Haerty W. Pathomorphological Aspects of the Heart in Septic Patients. In: Schlag G, Redl H. editors. Pathophysiology of Shock, Sepsis, and Organ Failure. Berlin: Springer, 1993:853-8.

8. Kubota H, Endo H, Noma M, et al. Xenopericardial roll graft replacement for infectious pseudoaneurysms and graft infections of the aorta. J Cardiothorac Surg 2015;10:133.

9. Slipczuk L, Codolosa JN, Davila CD, et al. Infective Endocarditis Epidemiology Over Five Decades: A Systematic Review. PLoS One 2013;8:e82665.

10. David TE, Gavra G, Feindel CM, et al. Surgical treatment of active infective endocarditis: a continued challenge. J Thorac Cardiovasc Surg 2007;133:144-9.

Cite this article as: Kubota H, Endo H, Ishii H, Minegishi S, Tsuchiya H, Takahashi Y, Funata S, Inaba Y. An infrared coagulator to assist surgical treatment of infective endocarditis. J Thorac Dis 2019;11(10):4364-4370. doi: 10.21037/jtd.2019.09.82 